\title{
Qualitative Risk of Falling Assessment Based on Gait Abnormalities
}

\author{
David Gagnon \\ Department of Computer Science \\ University of Quebec at Chicoutimi \\ Chicoutimi, Canada \\ David.Gagnon@uqac.ca
}

\author{
Bob-Antoine J. Menelas \\ Department of Computer Science \\ University of Quebec at Chicoutimi \\ Chicoutimi, Canada \\ bamenela@uqac.ca
}

\author{
Martin J.-D. Otis \\ Department of Applied Science \\ UQAC, REPARTI Center \\ Chicoutimi, Canada \\ Martin_Otis@uqac.ca
}

\begin{abstract}
Walking in an unfamiliar environment may include some risk of falling. For frail seniors, these risks can significantly increase according to their ability to maintain balance. Among several factors, the user's balance can be affected by several risks including the characteristics of the user's gait. To overcome this issue, this paper presents three methods: one using a statistical model, and two others using an artificial neural network (ANN). The latter two can be differentiated by the use of constraints applied onto the raw data. Centered on non-invasive augmented shoes, our proposed system uses mobile technology to provide an on-site assistance to users, replacing the bulky equipment usually needed for clinical gait analysis. The experimental framework is based on visual disturbances to induce variation in the parameters of the user's gait. Preliminary results obtained from this framework suggest that our models enable the risk level classification.
\end{abstract}

Index Terms-Risk of falling, gait analysis, visual perturbations

\section{INTRODUCTION}

It is known that elderly do face difficulties for mobility because of falling problems. Beyond the physical injuries, in many cases, falls leave a psychological impact due to the fear of falling. As a consequence, even without an injury, a fall can cause a loss in confidence and a reduction of mobility [7]. In spite of many remarkable achievements, fall prevention programs do not provide any real-time monitoring of daily activities of user. This have motivated conception of intelligent clothes that can detect potentially dangerous situations related to gait abnormalities.

For elderly, gait abnormalities may represent reduction of motor function resulting in an increased risk of falling. Indeed, the risk of falling can be a long-term feature related to mobility. However, a risk of falling gives no guarantees about forthcoming falls. It only gives an indicator about a dangerous situation that may lead to falls. Therefore, the risk analysis proposed in this paper is a qualitative evaluation where the risk is defined as a probability of falling between low (0) to very high (3) level. Those four risk levels are based on the risk analysis method described in [3]. In the latter, qualitative risk is assessed by combining different risk components. Each component is numerically rated to reflect a low to a high value. Then, the overall risk is obtained by combining them together.

In this paper, the device used for the computation of the risk of falling according to the user's gait is centred around an instrumented shoe, similar to these ones [13], [16], [18], driven by a real-time system running on a SmartPhone. The instrumented interface contains some force sensors and a variable bending resistor located inside a sole; which the latter is adapted to many types of footwear. Data measured from four FSR Force sensors and variable bending resistor (FlexSensor) are send via bluetooth wireless communication to a smartphone at a sampling rate of $100 \mathrm{~Hz}$. An Android application then implements the proposed algorithms in this paper to compute a risk of falling.

The contribution of our work is then the analysis of gait abnormalities by using three methods: one using a statistical model, and two others using an artificial neural network (ANN). These models are designed in order to qualitatively evaluate a risk of falling level while walking. The structure of the paper is organized into the following sections. First, a related works on the gait analysis using instrumented shoe is analysed. Second, three gait models for the risk of falling evaluation are described. Third, two experiments are detailed: the first one is used for the validation of the algorithms and the second one for the evaluation of their performances. Finally, a discussion on the algorithms performance shows a relation between gait abnormalities and risk level.

\section{RELATED WORKS}

Although many factors may increase the risk of falling, one of the most important is the user gait parameters variances. Indeed, Hamacher et al. concluded in [9] that linear variability of temporal measures of stride, swing and stance time are the parameters most capable of distinguishing between fallers and non-fallers. In order to compute these gait parameters, in [8], the authors used four gyroscopes located at lower limb for the analysis of gait asymmetry.

However, gait related issues are usually assessed with the help of instrumented shoes. Typically, those shoes uses sensors embedded in the sole to analyse the user gait. For instance, shoes with wireless capabilities have demonstrated the feasibility of computing walking parameters such as heelstrike, toe-off, foot orientation and position [5]. Also in [15], they computed an instability assessment model for health care professionals with the help of eight gait parameters, such as pressure correlation, step time, cadence and stance-to-swing 
ratio. Others also show interesting avenues about the gait anomalies detection and the gait phases recognition. For instance, the system proposed in [19] uses an instrumented shoe to detect different gait mode by phase-dependent pattern recognition for a below-knee amputee subject. Artificial intelligence techniques such as fuzzy logic and Hidden Markov Model were also use to detect gait patterns and to classify gait abnormalities [4], [11], [12]. However, despite the improvement in the gait analysis, none of these systems compute a qualitative risk of falling.

\section{GAIT ANALYSIS MODELS}

A step of the human gait is divided in two main phases: the stance phase, where the foot touches the ground, and the swing phase, where the foot leaves the ground. In this paper, the gait analysis is mainly done during the former phase. For this study, the stance phase is divided into three periods, as shown in Fig. 1: (1) the heel strike -the first heel contact with the ground-, (2) the midstance and (3) the toes-off -the propulsion phase of the gait-. In the following sections, steps will be timed according to those definitions. Thus, we refer the time pass since the last heel strike as the "current step time" and the midstance time portion as the "midstance time".

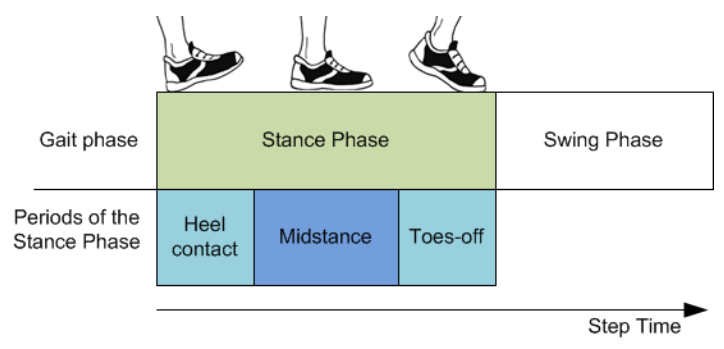

Fig. 1. Overview of gait phase

The main contribution of this work is centered on the comparison of three gait analysis models: (1) Statistical model (STAT), (2) Artificial neural network model using current data (ANN-RT) and (3) Artificial neural network model on data between a heel strike and toe-off (ANN-S). The former model is based on several statistics computed with extracted gait parameters from measured raw data. The two others methods, as their name implies, are based on artificial intelligence techniques. The main difference between both ANN models is the constraints applied on a temporal analysis and the features computed. The ANN-RT model computes current data - at a rate of $100 \mathrm{~Hz}-$, while the ANN-S relays on a windowing approach namely for each window, several gait features are computed.

Since the gait is specific to each user, these three models need a training stage prior to be used. This training process is indeed mandatory in order to take into account the physical and morphological differences between each walker and should be supervised by a clinician.

The STAT model, the ANN-RT model and the ANN-S model are further described in the following sections.

\section{A. Statistical Model}

Statistical models represent a first approach to artificial intelligence techniques. Further studies on the gait analysis use this model. Indeed, in order to characterize gait variability in patients with parkinson's disease, Wu and Krishnan proposed to use a statistical model [20]. Also, through an instrumented shoe, Noshadi et al. compute in real-time a variability index coming from gait parameters using statistical trends [15].

In our statistical model, the gait analysis is conducted by comparing several gait parameters with their receptive average value. The monitored parameters are obtained from data transmitted by the shoe and are divided according to footsteps. Thus, at each specified time from heel strike, the raw data is processed to obtained, for each FSR sensor, the beginning time, the pressure at current step time and the maximum pressure since the last heel strike. Moreover, the time taken for the stance phase and the swing phase are also monitored.

The mandatory training step takes a set of data from the usual gait of the user. Those data should come from supervised walking to ensure that the model is trained with a suitable gait, and of course, is not trained with unsteady gait. The parameters average value and their respective standard deviation are computed according equations (1) and (2):

$$
\begin{aligned}
\hat{D}_{j t} & =\frac{1}{|S|} \times \sum^{S}\left(D_{j t}\right) \\
\sigma_{j t} & =\frac{1}{|S|} \times \sqrt{\sum^{S}\left(\hat{D}_{j t}-D_{j t}\right)}
\end{aligned}
$$

where $D$ is the data of the parameter $j$ at time $t, \hat{D}$ the average and $\sigma$ the standard deviation. Sums are performed on all step samples $S$.

The three stages that composed the STAT gait analysis process are summarized in Fig. 2. At first, to extract risk of falling from current data $D$, the algorithm computes, for each selected parameter $j$ at time $t$ from the start of a heel strike, the ratio $R_{j t}$ of how many standard deviation the value is from the average $\hat{D}_{j t}$ as seen in (3). Thereafter, each ratio $R_{j t}$ is then summed together after being multiplied by a weight $W_{j}$. The result computed by (4) gives an indicator $G$ at time $t$ of the gait abnormalities. The global indicator $G$ is obtained by summing those timed indicators according to (5). Finally, the third stage discretizes the risk by applying thresholds to the global indicator.

$$
\begin{aligned}
R_{j t} & =\left(D_{j}-\hat{D}_{j t}\right) / \sigma_{j t} \\
G_{t} & =\sum_{j}\left(W_{j} \times R_{j t}\right) \\
G & =\sum_{t}\left(G_{t}\right)
\end{aligned}
$$

In fact, if current data are closed to the computed average data, the global indicator will tend to zero and the risk will 


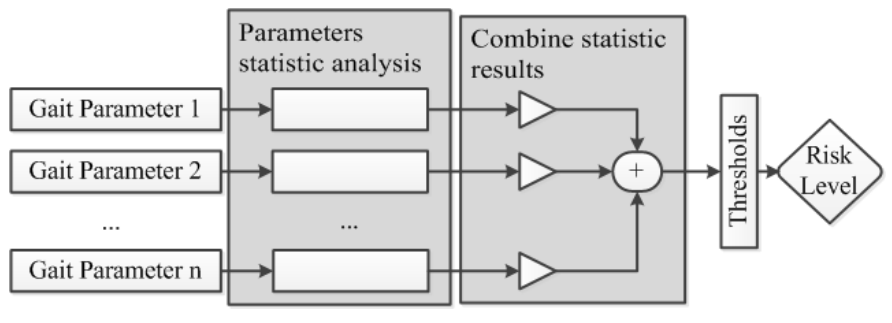

Fig. 2. Analysis process of the walking data

be low. Otherwise, the indicator will grow and the risk will increase, thus giving real-time indicator of gait abnormality.

\section{B. Artificial neural network model ANN-RT}

As its name implies, the Artificial neural network ANNRT model analyses the gait with current input data. Such methods is used for distinguishing gait pattern between normal healthy subjects and Parkinson Disease (PD) patients [10]. In a similar manner, it allows identification of abnormal gait pattern in Parkinson's disease subjects during normal walking [14]. The ANN-RT model does not need a full step (between heel strike to toe-off) to compute the risk. However, raw data cannot be used as input directly for the ANN-RT models. Therefore a pre-processing stage is required in order to extract gait features that will be used as inputs of the artificial neural network. As a result, the ANN-RT model is implemented with three computation stages: features extraction, artificial neural networks computation and risk discretization as shown in Fig. 3.

Features used include bending of the sole, the pressure ratio at heel position $\left(R_{t}\right)$, the pressure ratio at toe positions $\left(R_{t}\right)$ and the midstance time ratio $\left(R_{m s}\right)$. Though, the raw data coming from bending of the sole are directly used as a feature, the three others are pre-processed. For this, equations (6) and (7) are used to extract the two pressure ratio features from sensors $(P)$. To compute $R_{m s}$, the current midstance time is divided by the current step time as shown in (8):

$$
\begin{aligned}
R_{h} & =\left(P_{h i}\right) /\left(P_{h i}+P_{h e}\right) \\
R_{t} & =\left(P_{t i}\right) /\left(P_{t i}+P_{t e}\right) \\
R_{m s} & =T_{m s} / T_{\text {step }}
\end{aligned}
$$

where the index represents the sensor locations: heel $(h)$, toes $(t)$, interior $(i)$, and exterior $(e)$ of the sole.

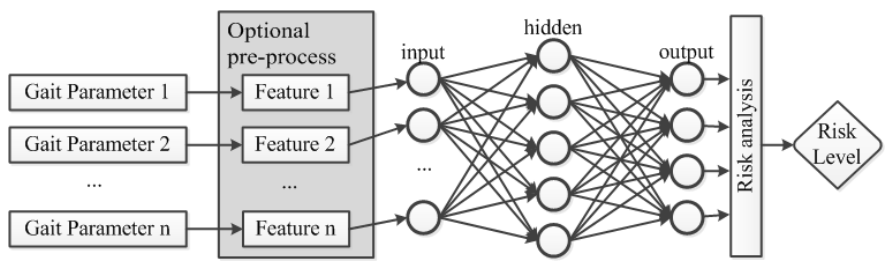

Fig. 3. Artificial Neural Network Model

The artificial neural network is composed of four inputs, four outputs and a hidden layer of eight neurones. Pre- processed features are used as input, while the outputs reflect the risk according to the user gait.

To discretize the risk, the four neurone output values are weighted and summed together using 9:

$$
\text { Risk }=\sum\left(O_{k} \times w_{k}\right)
$$

where $O$ is the output value of the neurone $k$ and $w$ its according weight. As the STAT model, thresholds are then applied to obtain the associated risk.

Though, the gait analysis is straight forward, ANNs needs training to process data accordingly. Particularly, the ANNRT model is trained using the Backpropagation algorithm and require a proper training data set. This set should contained step samples of all the four risk levels. To get those, several step are captured and then analysed with the STAT model. Thereafter, the training set is obtained by the selection of some data from each rated step. Since unsteady gaits are necessary for training, those should be acquired with the help of a clinician.

\section{Artificial neural network model applied to step data}

The ANN-S model is very similar to the ANN-RT one. One may refer to Fig. 3 for an overview of the three process stages: features extraction, artificial neural networks computation and risk discretization.

The main difference between ANN-S and ANN-RT models resides in the features selection. In the former, features are not dependent of a full step. In the latter, features are extracted only when a step is completed. Those include the maximum foot bending in the step, the midstance time ratio of a step as computed using (8), the average pressure during midstance $P_{m s}$ and the stance-to-swing ratio $R_{\text {StanceToSwing }}$. The two last features are computed according to (10) and (11):

$$
\begin{aligned}
P_{m s} & =\sum_{\text {stance }} /\left(P_{j t} / T_{m s}\right) \\
\left.R_{\text {StanceToSwing }}+T_{\text {stance }}\right) & \left.=T_{\text {sto }}\right)
\end{aligned}
$$

where $P$ is the pressure obtained from sensor $i$ at a time $t$ and $T_{m s}$ is the midstance time. $T_{\text {stance }}$ and $T_{\text {swing }}$ represent respectively the total time of the stance (foot on the ground) and the swing (foot in the air) according to the current step.

As the ANN-RT model, the artificial neural network of the ANN-S model is composed of four inputs, four outputs and a hidden layer of eight neurones. In the same way, the training data set is obtained from a selection of several steps analysed with the STAT model. This paper proposes to use the same training set for both ANN models.

\section{VALIDATION AND PERFORMANCE}

In order to validate the three algorithms presented in this work, two experiments are conducted. The first one assesses the ability of proposed algorithms to detect and classify abnormalities in gait. The second one compares the algorithms performance to analyse gait influenced by three visual perturbations. 


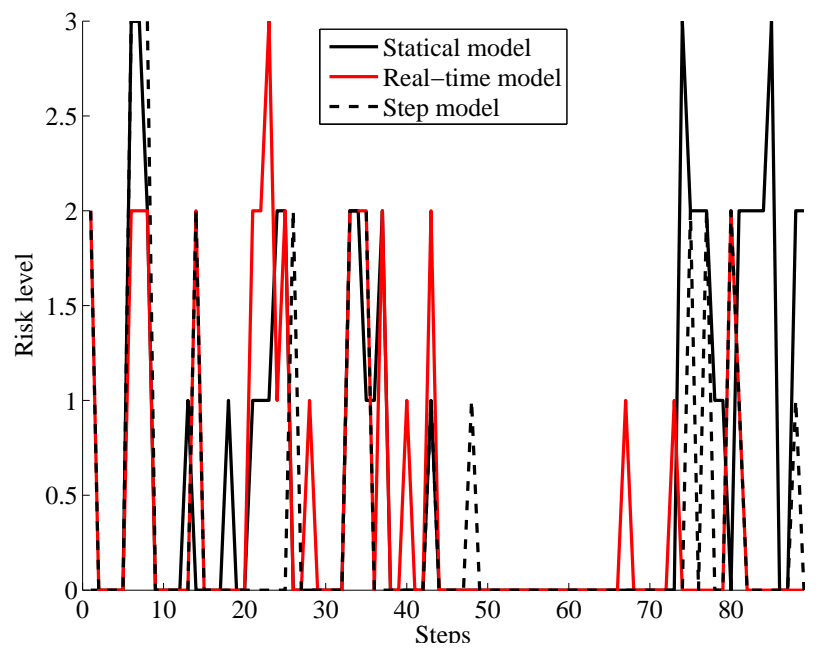

Fig. 4. Summary of the computed risk's according to each step for the STAT model (black curve), the ANN-RT model (red curve) and the ANN-S model (dot curve).

\section{A. Detection and classification of gait abnormalities}

The goal of the first experiment is to evaluate the ability of the three models to differentiate and quantify gait abnormalities. In fact, this is a preliminary stage to evaluate algorithms performance. To do so, a gait sequence is analysed through the three algorithms and results are graphically analysed. To be usable in the second experiment (visual perturbations), the three algorithms should output similar risk evaluations.

Data is collected from a healthy subject walking at average speed. Around ninety strides were taken to capture the gait parameters. The participant was asked to walk along a corridor while varying its gait between a normal gait, a limping gait, a balancing gait and a toes first gait. In fact, most of the gait variation asked included a major change in the user's gait phase timing. About a third of the steps were considered as abnormal compared to the participant standard gait.

Fig. 4 presents a summary of the risk computed by the algorithms according to each step recorded. Each risks is represented by a number from zero (low risk) to three (very high risk). In Fig. 4, the black curve shows the statical model results while the red and dot curves respectively show the ANN-RT and the ANN-S outputs. As a first observation, one notes a correspondence between the ANN-S and the STAT model evaluations. On the other hand, the ANN-RT model seems to diverge from the two others. However, by looking at summary table I, the algorithms have found around the same risk level's number.

\begin{tabular}{cccc}
\hline \hline Risk level & STAT\#1 & ANN-RT\#2 & ANN-S\#3 \\
\hline 0 & 60 & 68 & 73 \\
1 & 10 & 6 & 4 \\
2 & 15 & 14 & 9 \\
3 & 4 & 1 & 3
\end{tabular}

TABLE I

SUMMARY TABLE OF THE MODEL COMPUTED RISK'S
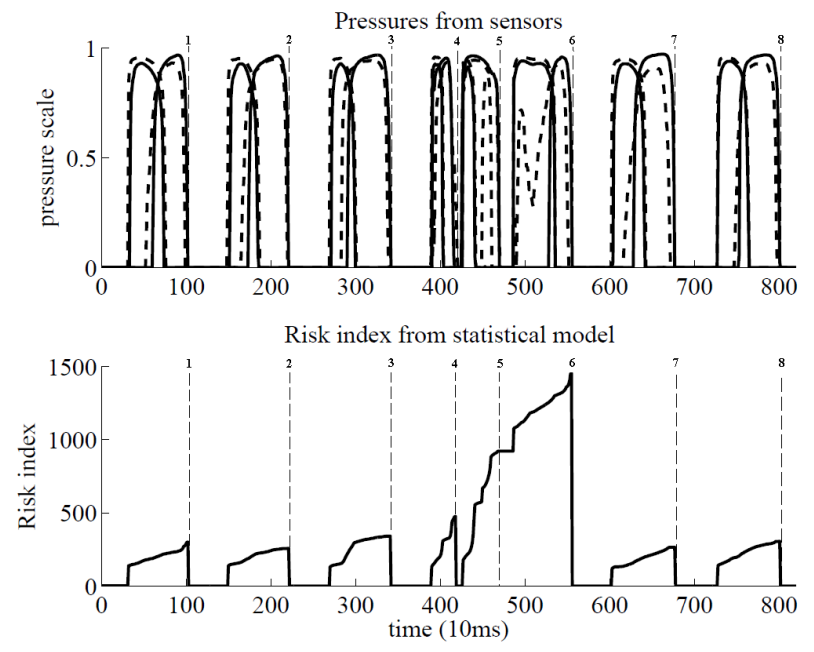

Fig. 5. Pressures from the four sensors under the foot (top) and risk analysis of the STAT model (bottom)
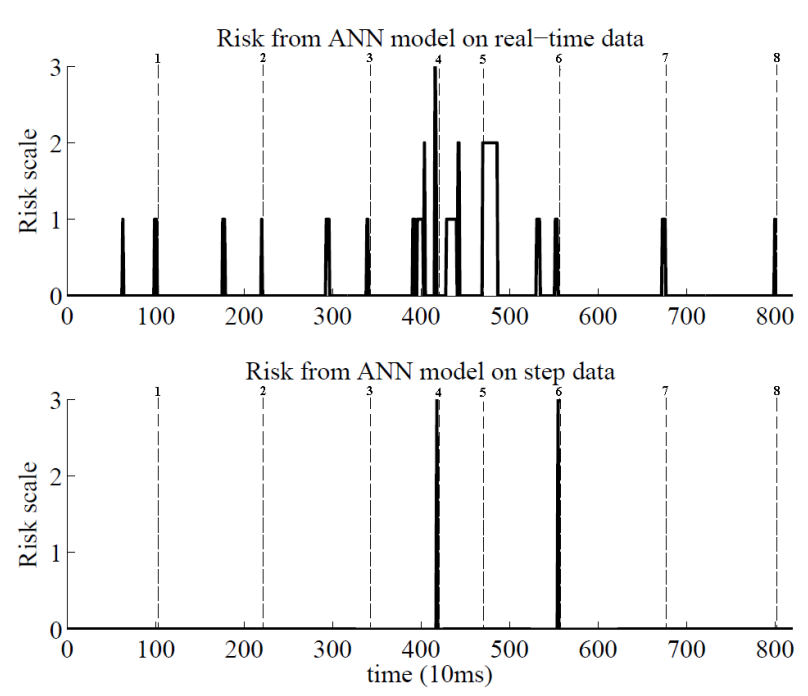

Fig. 6. Risk analysis of the ANN-RT model (top) and the ANN-S model (bottom)

As an example, four different representations of eight walking steps sequence are shown according to time. Fig. 5 shows raw pressures from FSR sensors (top) and STAT model analysis prior to the threshold stage (bottom) while the Fig. 6 shows the ANN-RT (top) and ANN-S (bottom) analysed risks. From the pressure curve, one may find step numbers four, five and six different from the five others. Accordingly, the statical model shows a significant difference in the gait parameters for those three steps. ANN models also detect abnormalities for these steps. On the other hand, the ANN-RT one shows medium risk (risk level one) for most of the steps but detects variations for the fourth, the fifth and the sixth step. The ANN$\mathrm{S}$ algorithm only detects two very high risk levels on step four and six while other steps are evaluated as low risk level. 


\section{B. Performance evaluation}

In order to evaluate the performance of the algorithms, the models should be tested in a more realistic situation. Since older population couldn't be used at this stage of the project, the modification of visual acuity was used to induce abnormal gaits. Indeed, the risk of falling increases with poor depth perception/stereo-acuity and poor low-contrast visual acuity [17].

Moreover, several studies demonstrated the relation between gait changes (or postural stability) and visual perturbation in the young and older age groups. Experiments were performed using participants with no history of falls and normal vision. The visual stimulus was mainly lens magnification [6], binocular refractive blur [1] and cataract simulation [2].

Knowing these previous studies, we developed an experiment using three modified pairs of glasses in order to simulate four risk levels. For example, an increase of visual perturbation will increase respectively unsteady steps or gait abnormalities. Indeed, four visual conditions were explored: no visual disturbance $(\mathrm{C} 0)$, lightly obscured vision $(\mathrm{C} 1)$, obscured vision $(\mathrm{C} 2)$ and highly obscured vision (C3). Thereby, it is expected that our three models should differentiate risk level (related to gait abnormalities) according to visual disturbances.

Four participants take part in this experiment (labelled as P1, P2, P3 and P4 in the following). All participants were graduated students or university staff aged between 23 and 36 years old. Among the participants, none have stated any particular gait issues. The participants were asked to walk along a corridor in a straight line four times; each times they are wearing different glasses (or no glasses). During the tests, an auditory disturbance was applied to prevent the influence of heel strike sounds on the user gait.

For each participant, the data analysis is done in the following processing stage. All acquired data are first analysed according to the three algorithms. However, no threshold is yet applied; the models outputs at this stage are only risk indexes. Then, for each risk, results are merged, sorted and equally divided in four subgroups representing a different risk level from which three thresholds are extracted. Once done, each risk index is then discretized, thus giving, for each visual condition ( $\mathrm{C} 0$ to $\mathrm{C} 3$ ), the risk level for each step. For each visual disturbance, the average risk is then computed according to each model and each participant.

A summary of the results are shown in Fig. 7. First, for the gait analyzed by the STAT model, participants P1 and P2 show an increasing risk following the visual disturbances while participant P3 has a slight risk decrease for the $\mathrm{C} 2$ condition. Although the forth participant do not show a monotonic increase of the risks, there is still a difference in the gait analysis between no visual perturbations and the three other conditions. Second, the results coming from the ANN-RT model are strictly increasing for P1, P3 and P4. The evaluation of participant P2's step detects a quite difference between $\mathrm{C} 0$ condition and the three others. Finally, the results given by the ANN-S model show a corresponding risk increase according to visual perturbation augmentation for each of the participant.

\section{Discussion}

In the first experiment, the three models were able to detect abnormal gait. By comparing the ANN models with the statistical one, similar rates of $76.6 \%$ and $75.28 \%$ for respectively the ANN-RT and the ANN-S are obtained. Those high rates indicate that the three models achieve similar performance and give about the same risks. However, a closer look in the Fig. 4 shows that ANN-RT model seems less accurate. Particularly, it tends to evaluate higher risks than the two other models. Still, the algorithms detect and classify risk levels for some important variations in the gait parameters. Although only one participant takes part in the first experiment, some conclusions on the three models can still be drawn. Indeed, since the algorithms are trained according to each user, similar results should be obtained for other user if gait parameter variations are found. Of course, in real life situations, gait parameter variations are less noticeable than those evaluated in the first experiment. However, by using visual disturbance to induce variations in the gait, the conditions of the second experiment are closer to natural events and can then be differentiated by the ANN.

Results obtained for the second experiment show that, for most of the participants, the three models were able to detect an increase risk level when visual disturbance were augmented. Though, it seems that models do not always detect a risk level increase. For instance, the participant P4's risk evaluations by the STAT model for condition $\mathrm{C} 1, \mathrm{C} 2$ and $\mathrm{C} 3$ do not spotted the expected risk augmentation. Similar results can be seen in ANN-RT model for the participant P2. Thereby, what conclusion can be drawn from those observations?

First of all, one has to note that, even if visual perturbations tend to increase the gait variability, their effects are only probable. In other word, one can have no significant change in their gait while wearing the different glasses. Moreover, nothing prevents a user without visual disturbance to occasionally make an unusual step due to external perturbations or uncontrolled variables. Thus, it is expected that for some step sequences, computed average risks do not reflects the according visual condition.

Secondly, the three algorithms use different features to evaluated the risk level; the STAT uses several timed parameters, the ANN-RT uses online data and the ANN-S uses processed data once the step is completed. Thus, even if features compositions are similar, difference in the processed risk may occur. This is particularly visible in the results given by the ANN-RT model of the first experiments, where it detects different risks compared to others.

Finally, it is known that our brain have a great adaptability. Since the results of the second experiment were obtained by increasing visual disturbances, it is possible that some participants got used to the visual perturbations and unconsciously corrected their gait. This could explain conflicting results for the $\mathrm{C} 3$ condition.

Despite multiples uncontrolled variables from human factors, results obtained did show, for most of the participant, a corresponding increase of the average risks when a decrease 

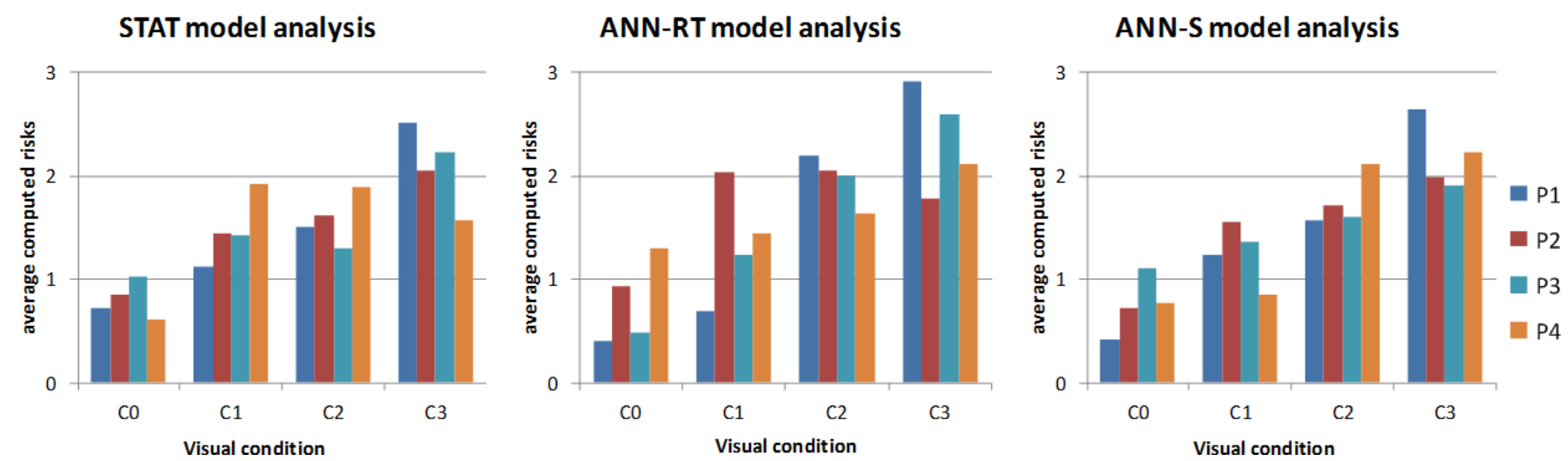

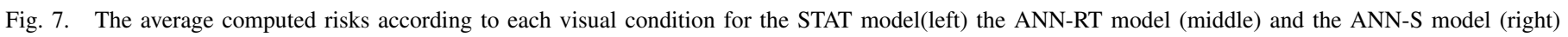

visual perception was applied. Consequently, the three models proved their ability to classify risk levels according to the user gait. By comparing the three models, it seems that the ANN$\mathrm{S}$ model gives better results than the two others. Also, the STAT model shows good results from most participants. Thus, statistic model for the gait analysis has proved good efficacy. Finally, the ANN-RT model gives satisfactory results but is less accurate than the two others.

\section{CONCLUSION AND FUTURE WORKS}

This paper has presented three models in order to analyse qualitatively a risk level related to gait abnormalities. The three proposed models are respectively based on statistics, on artificial neural network on real-time acquired data and on artificial neural network on data of a complete step. Models were evaluated by two experiments. The first one validated the ability of the three models to detect and classify risk level when voluntary gait variations were induced. The second experiment compared and validated the gait analysis by inducing gait variation through visual disturbance. Despite of some human factors, the three algorithms are able to evaluate some gait abnormalities and give accordingly a risk level.

Knowing the according risk level, on-site assistance can be provided to frequent fallers. To do so, future works will use the computed risk level in order to warn the user through vibrotactile feedbacks. Moreover, by including several risk detections with the designed instrumented shoe described in this paper, the whole systems could be used as comprehensive mobile assistance tool for elderly population.

\section{REFERENCES}

[1] V. Anand, J. Buckley, A. Scally, and D. Elliott. The effect of refractive blur on postural stability. Ophthalmic and Physiological Optics, 22(6):528-534, 2002.

[2] V. Anand, J. Buckley, A. Scally, and D. Elliott. Postural stability changes in the elderly with cataract simulation and refractive blur. Investigative Ophthalmology and Visual Science, 44(11):4670-4675, 2003.

[3] L. Anthony. Qualitative and quantitative risk analysis. In Quantitative Health Risk Analysis Methods, volume 82 of Int. Series in Operations Research \& Management Sci., pages 1-35. Springer, 2006.

[4] J. Bae and M. Tomizuka. Gait phase analysis based on a hidden markov model. Mechatronics, 21(6):961-970, 2011.
[5] S. Bamberg, A. Benbasat, D. Scarborough, D. Krebs, and J. Paradiso. Gait analysis using a shoe-integrated wireless sensor system. IEEE Tr. on Information Technology in Biomedicine, 12(4):413-423, 2008.

[6] G. Chapman, A. Scally, and D. Elliott. Adaptive gait changes in older people due to lens magnification. Ophthalmic and Physiological Optics, 31(3):311-317, 2011.

[7] D. Ganz, Y. Bao, P. Shekelle, and L. Rubenstein. Will my patient fall? Journal of the American Medical Association, 297(1):77-86, 2007.

[8] D. Gouwanda and S. Arosha Senanayake. Identifying gait asymmetry using gyroscopes-a cross-correlation and normalized symmetry index approach. Journal of Biomechanics, 44(5):972-978, 2011.

[9] D. Hamacher, N. Singh, J. Van Dieen, M. Heller, and W. Taylor. Kinematic measures for assessing gait stability in elderly individuals: A systematic review. J. of the Royal Society Int., 8(65):1682-1698, 2011.

[10] H. Hazfiza Manap, N. Md Tahir, A. I. M. Yassin, and R. Abdullah. Anomaly gait classification of parkinson disease based on ann. In IEEE Int. Conf. on System Eng. and Tech., ICSET, pages 5-9, 2011.

[11] K. Kong, J. Bae, and M. Tomizuka. Detection of abnormalities in a human gait using smart shoes. In M. Tomizuka, editor, Sensors and Smart Structures Technologies for Civil, Mechanical, and Aerospace Systems, pages 69322G-69322G-10. SPIE, 2008.

[12] K. Kong and M. Tomizuka. Estimation of abnormalities in a human gait using sensor-embedded shoes. In IEEE/ASME International Conference on Advanced Intelligent Mechatronics, AIM, pages 1331-1336, 2008.

[13] M. Magana and R. Velazquez. On-shoe tactile display. In IEEE Int. Workshop on Haptic Audio VE and Games, pages 114-119, 2008.

[14] H. Manap, N. Md Tahir, and A. Yassin. Statistical analysis of parkinson disease gait classification using artificial neural network. In IEEE Int. Sym. on Signal Processing and Inf. Tech., pages 060-065, 2011.

[15] H. Noshadi, S. Ahmadian, H. Hagopian, J. Woodbridge, F. Dabiri, N. Amini, M. Sarrafzadeh, and N. Terrafranca. Hermes: Mobile balance and instability assessment system. In Conference on Bio-inpsired Systems and Signal, BIOSIGNALS, pages 264-270, Jan. 2010.

[16] M. J.-D. Otis and B.-A. J. Menelas. Toward an augmented shoe for preventing falls related to physical conditions of the soil. In IEEE Int. Conf. on Systems, Man, and Cybernetics, pages 1-6, 2012.

[17] L. Salonen and S.-L. Kivela. Eye diseases and impaired vision as possible risk factors for recurrent falls in the aged: A systematic review. Current Gerontology and Geriatrics Research, 2012, 2012.

[18] J.-S. Shieh, B. C. Jiang, K.-H. Wang, and W.-H. Yang. Fall-risk evaluation and balance stability enhancement system and method. US Patent 2011/0251520 A1, 2011.

[19] X. Wang, Q. Wang, E. Zheng, K. Wei, and L. Wang. A wearable plantar pressure measurement system: Design specifications and first experiments with an amputee. Advances in Intelligent Systems and Computing, 194 AISC(VOL. 2):273-281, 2013.

[20] Y. Wu and S. Krishnan. Statistical analysis of gait rhythm in patients with parkinson's disease. IEEE Tr. on Neural Systems and Rehabilitation Eng., 18(2):150-158, 2010. 